\title{
Coupling and Shielding Properties of the Baffle in ICP System
}

\author{
Jozef Brcka ${ }^{1}$ and R. Lee Robison ${ }^{2}$ \\ ${ }^{1}$ Technology Development Center, TEL US Holdings Inc., Suite 244, 255 Fuller Road, Albany, NY 12203, USA \\ ${ }^{2}$ TEL Technology Center America LLC, Suite 244, 255 Fuller Road, Albany, NY 12203, USA
}

Correspondence should be addressed to Jozef Brcka, jozef.brcka@us.tel.com

Received 28 October 2010; Accepted 13 June 2011

Academic Editor: Agostino Bruzzone

Copyright (C) 2011 J. Brcka and R. L. Robison. This is an open access article distributed under the Creative Commons Attribution License, which permits unrestricted use, distribution, and reproduction in any medium, provided the original work is properly cited.

This contribution is dealing with experimental and computational evaluation of the deposition baffle that is transparent to radio frequency (RF) magnetic fields generated by an external antenna in an inductively coupled plasma (ICP) source but opaque to the deposition of the metal onto a dielectric wall in ionized physical vapor deposition (IPVD) system. Various engineering aspects related to the deposition baffle are discussed. Among the many requirements focus is on specific structure of the slots and analysis to minimize deposition on the baffle (we used a string model for simulating the profile evolution) and deposition through the DB on dielectric components of the ICP source. Transparency of the baffle to RF magnetic fields is computed using a three-dimensional (3D) electromagnetic field solver. A simple two-dimensional sheath model is used to understand plasma interactions with the DB slot structure. Performance and possible failure of device are briefly discussed.

\section{Introduction}

Many ICP sources require a protecting shield from the plasma. Typical example is an ionized physical vapor deposition (IPVD) which is used for metallization process in IC fabrication. The high-density plasma generated by an ICP source provides the high-density ionized metal fluxes that are important for high-aspect ratio feature metallization and seed layer deposition in IC fabrication. The fundamentals of IPVD technology have been described in publications elsewhere, for instance [1-5]. In this paper, we will focus on the physics and engineering aspects of an RF transparent baffle but opaque to deposition in experimental IPVD system $[6,7]$ with an external ICP antenna. In Section 2, we will describe basic properties of the ICP with deposition baffle (DB). In Section 3, we will deal with the most important criteria and performance of the deposition baffle.

\section{ICP with Metallic Baffle}

The IPVD technique is widely used for the deposition of metallic films. Many IPVD systems employ an ICP source to create high-density metal-containing plasma. Here, the IPVD apparatus under investigation is described in more detail in [8]. The cross-section of such ionized metal source is shown in Figure 1. It consists of the source of metal species (a conical annular target biased by negative DC potential) that is axially positioned with planar high-density ICP source. When depositing, species from the plasma create a significant source of contamination on the RF-transparent dielectric window. Specially, due to the conductive origin of the contamination, the efficiency of the ICP system can be strongly diminished, and the potential risk for hardware or instrumentation failure is highly possible in such system. Consequently ICP source shielding has to be used.

Contrary to configurations published by other researchers [9-13], we are using an external antenna $[6,14]$ to couple $13.56 \mathrm{MHz}$ RF generator to the plasma. This antenna generates on-axis peaked RF power density distribution with maximum up to $30 \mathrm{~W} \mathrm{~cm}^{-2}$ [6]. The $\mathrm{RF}$ magnetic field is strongly compressed when passing through slots of the deposition baffle to couple to the plasma. This generates strong induced currents in the plasma and produces a high density of the charged species. Probe measurements [7] in such a system determined an electron density up to $\sim 5 \times 10^{12} \mathrm{~cm}^{-3}$ within much larger bulk plasma volume than in typical PVD systems. We want to mention that such density is achieved without exposure of the external magnetic field to the core plasma. Such high-density plasma provides 


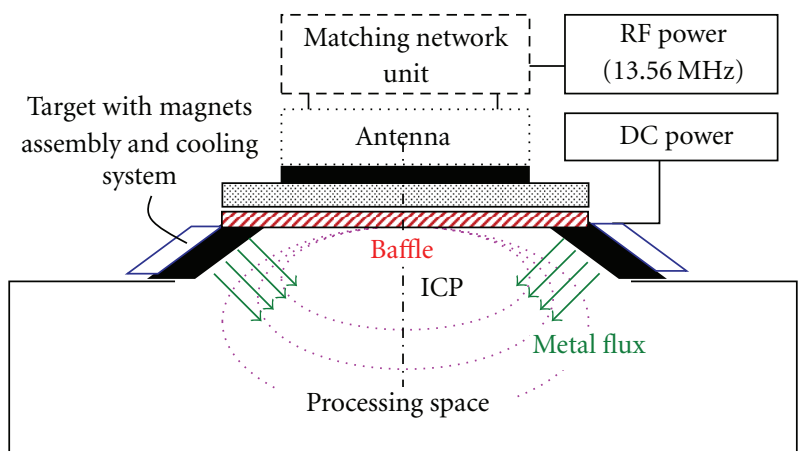

FIGURE 1: Cross-section of the IPVD system with annular conical target and high-density inductively coupled plasma (ICP) source.

high-density ionized metal fluxes that are important in highaspect ratio feature metallization and seed layer deposition in IC fabrication.

At these conditions, reasonable deposition rates are achieved using only low DC power at the target $\left(2-3 \mathrm{~W} / \mathrm{cm}^{2}\right)$. For example, deposition rates were about $80-200 \mathrm{~nm} / \mathrm{min}$ for various customized magnetic field assemblies to confine electrons closely to target and with an excellent repeatability and deposition uniformity on the $300 \mathrm{~mm}$ wafers with typical pressure range from several tenths of Pascal to about $15 \mathrm{~Pa}$ [15]. For comparison, conventional PVD tools [16] consume about $\sim 10 \mathrm{~W} / \mathrm{cm}^{2}$ power at the cathode providing plasma with magnetically confined electron density about $10^{11} \mathrm{~cm}^{-3}$ closely to the target. Efficient coupling of the RF magnetic field from the antenna is crucial for such performance. We will discuss some of these requirements in the Section 3 below.

\section{Properties and Performance}

The dielectric window serves as a barrier between the ICP plasma processing zone and atmospheric pressure. Obviously, when depositing metal on a substrate, it is necessary to avoid depositing metal on the dielectric window by using a suitable deposition baffle. The DB in the IPVD tool, has several functions and has to satisfy many requirements. Major requirements are imposed on the following items:

(1) to provide excellent shielding performance against contamination fluxes,

(2) to allow efficient inductive coupling of the RF power from the ICP antenna to the plasma,

(3) to screen out the plasma from a capacitive E field of the antenna,

(4) to provide a grounding potential at internal surface opposing either to the antenna or plasma,

(5) to enable and sustain plasma ignition through its body,

(6) to provide thermal control of the interior walls,

(7) to provide a robust support of the dielectric window, and

(8) to be reliable hardware part over its lifetime.
3.1. Shielding Properties. As we mentioned, the DB is used to protect an internal surface of the dielectric window from conductive metallic deposits. We have developed $[17,18]$ a planar, high-cooled version with "bridged slots" for lowcontamination performance at the dielectric window. Simplified geometry is illustrated by "chevron" shape obvious from cross-section illustrated in Figure 2. Deposition of the metal on such structure is evaluated by modified 2D profile simulation [19] in Figures 2(a), 2(b), and 2(c) for different ion-to-neutral flux ratios. The neutral metal flux is providing isotropic deposition, and the metal ion flux is directional and generates also redeposition due to a sputter etch back on the DB structure. Figure 2 shows simulated deposition profiles of a $\mathrm{Cu}$ coating on the $\mathrm{DB}$ over the period five times longer than its life time. In Figure 2(a), neutral flux only is assumed $(0 \% \mathrm{Cu}$ ionization) with no sputter etch back, and simulation is sustained up to a film thickness of $5 \mathrm{~mm}$ at the flat field. Figure 2(b) shows the neutrals flux and ions flux (50\% Cu ionization), 10\% Ar sputter etch back, and/or $\mathrm{Cu}$ self-sputtering. Increased copper ionization (85\%) and $15 \%$ Ar sputter etch back and/or Cu self-sputtering is shown in Figure 2(c). From simulation results, we observed that increased ion-to-neutrals ratio sustains the "chevron" structure transparent enough to RF magnetic fields. Thus, sustaining high-density plasma (ICP) naturally provides the slots are not closed by deposited material over the lifetime of the target. The actual deposition of $\mathrm{Cu}$ coating on the DB having chevron slots over approximately a half-lifetime operation is shown in Figure 2(d).

There are feasible several candidates of DB with different slot structure geometries (see, Figure 3). So-called double-L structure (2L) in Figure 3(a), " $Z$-slot" structure (Figure 3(b)), and double-L structure with cavity (2LC slot) in Figure 3(c) were examined. The deposition opacity of these structures was validated by Monte-Carlo simulation [20] and exhibited high opacity to the deposition flux.

More slot structures were tested both by simulation and experimentally. For example, the structure shown in Figure 4(a) - "chevron with blades" — had very good deposition opacity (an actual structure machined in the DB is illustrated in Figure 4(b)). A silicon wafer placed immediately behind the DB and exposed to a cumulative deposition of copper exhibited residual deposition (Figure 4(c)) only after many hours of operation. An estimate of metal thickness deposited on wafer using resistivity values of the film is given in Figure 4(d). Nevertheless, of high opacity to deposition, this type of slot structure had tendency to fail (see Section 3.4 below) due to the disproportional heat load on individual slots as we found out later by heat transfer simulation and experimental observation. The investigations of more specific slots configurations were discussed yet in $[6,7]$.

3.2. Efficient Inductive Coupling and Plasma Ignition. Described above slot structures (Section 3.1) were tested and showed efficient RF magnetic field transparency. The 3D electromagnetic simulations were computed by commercial engineering software [21]. Figure 5 shows the distribution of the RF electromagnetic energy density at the exit from individual slots. All slots under investigation have comparable 


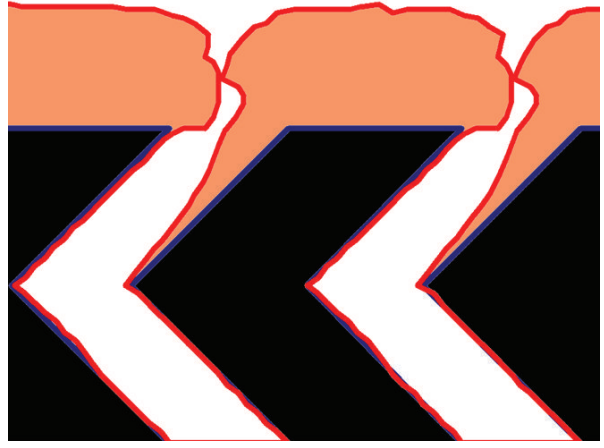

(a)

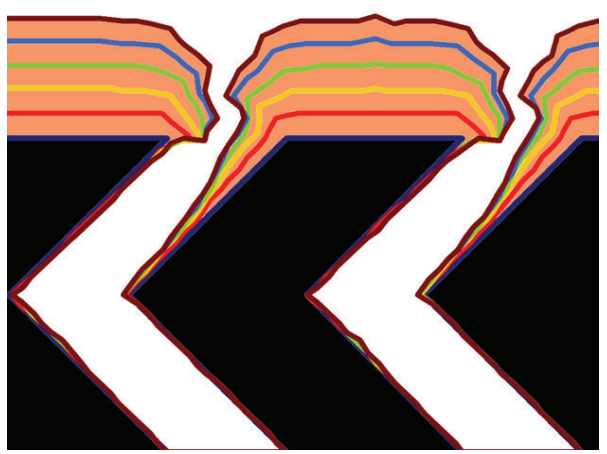

(c)

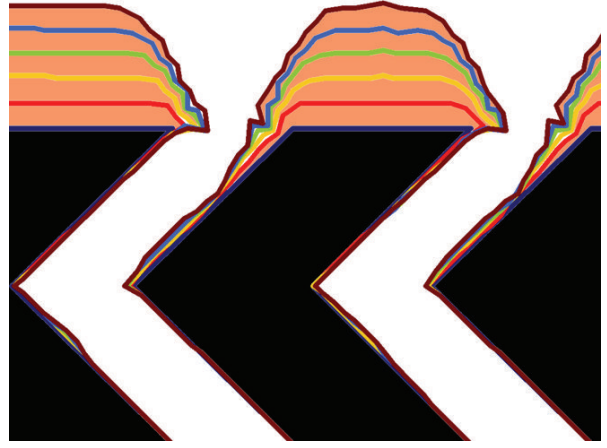

(b)

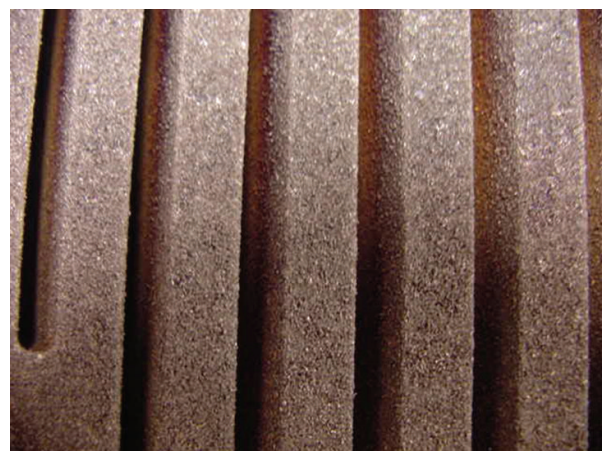

(d)

FIGURE 2: Simulated deposition profile of the Cu coating on the deposition baffle over the period five times longer than its lifetime: (a) assumed neutrals flux only ( $0 \% \mathrm{Cu}$ ionization) with no sputter etch back, the simulation was sustained up to thickness 5 mm at the flat field; (b) the neutrals flux and ions flux (50\% Cu ionization), assumed 10\% Ar sputter etch back; and (c) neutrals flux and ions flux (85\% Cu ionization), 15\% Ar sputter etch back. Photo (d) illustrates the actual deposition over the half lifetime of the deposition baffle.

Metal-containing plasma

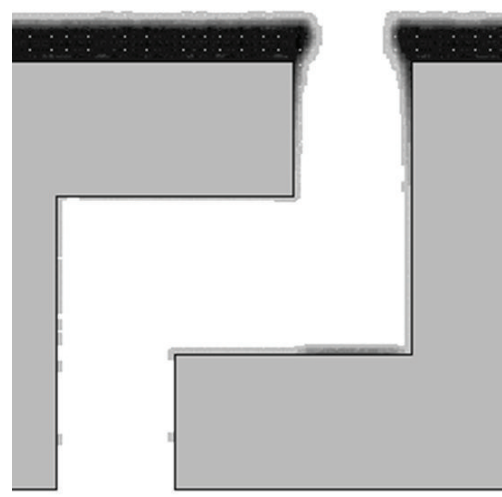

(a)

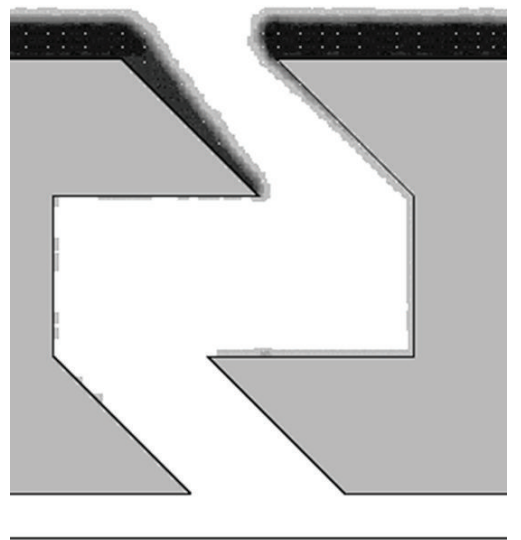

(b)

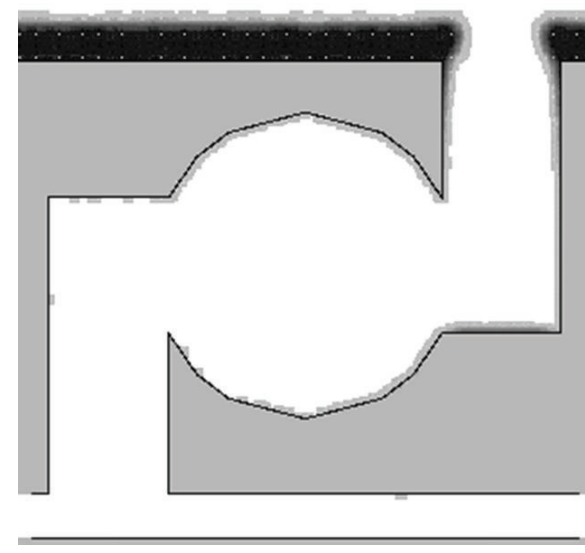

(c)

Figure 3: The deposition profile and opacity of the DB with various slots determined by Monte-Carlo simulation: (a) is double-L structure (2L), (b) structure of " $Z$-slot", and (c) double-L structure with cavity (2LC slot).

transparency to RF magnetic fields, however, more compact slot structures are preferable due to increased efficiency per unit area and higher-dimensional variability in design. The $E-H$ transition occurs in the 200-600 W interval, achieving similar plasma density values for all slot types saturating up to $4-5 \times 10^{12} \mathrm{~cm}^{-3}$ [7]. The DB implementation affected the $\mathrm{E}-\mathrm{H}$ transition (a hysteresis shape). The hysteresis for ICP excitation without DB was much wider than hysteresis when using DB. However, low RF power plasma heating was more efficient with DB (versus the case without incorporated DB) 


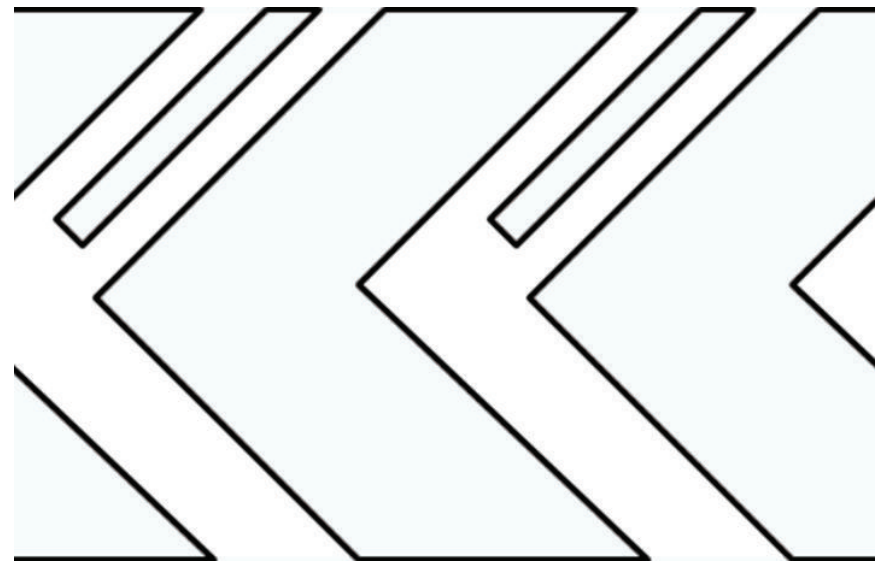

(a)

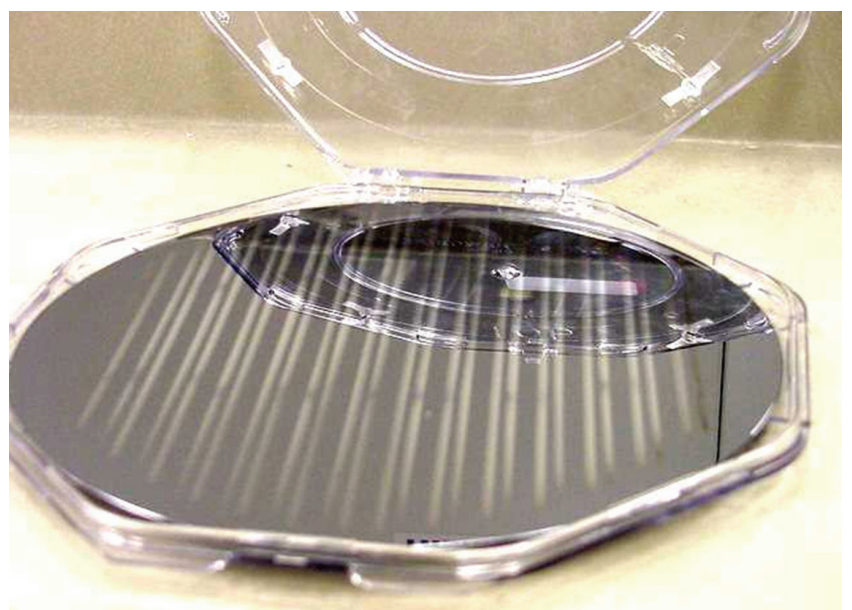

(c)

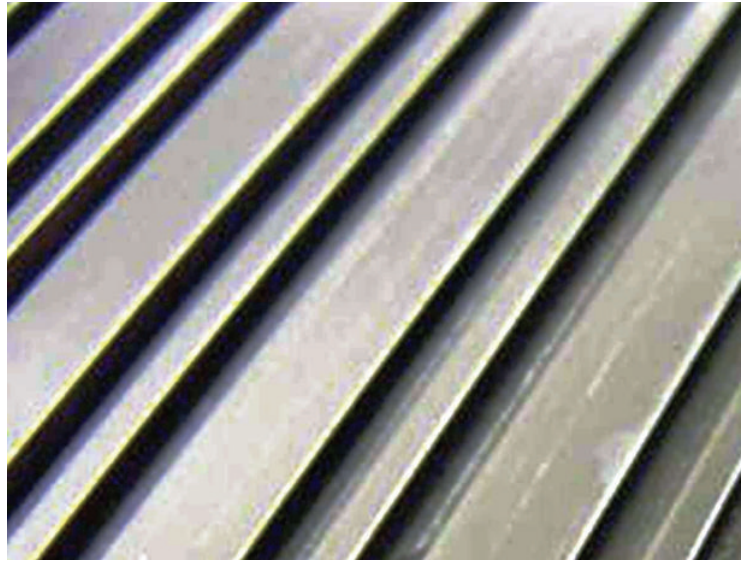

(b)

$\mathrm{Cu}$ film thickness on ceramic window

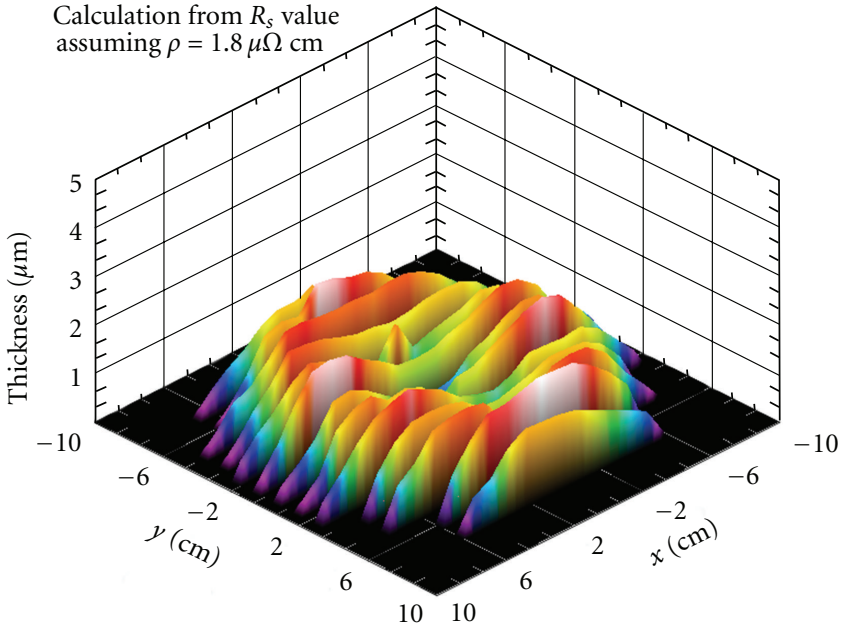

(d)

Figure 4: Chevron slot structure combined with thin blade: (a) cross-section of the structure, (b) actual photo of clean deposition baffleside exposed to the metal containing plasma, (c) cumulative deposit traces on the testing wafer, and (d) actual profile and thickness of the cumulative deposition on the wafer (calculated from sheet resistance of the metallic film).

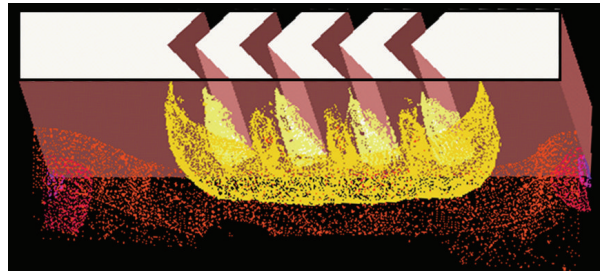

(a)

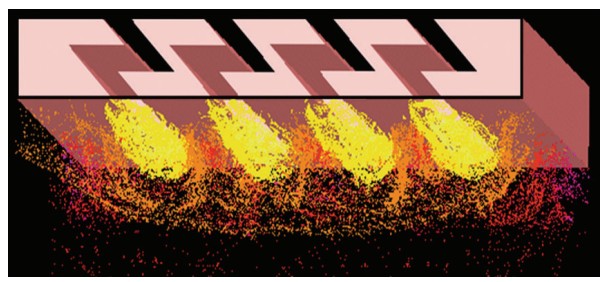

(c)

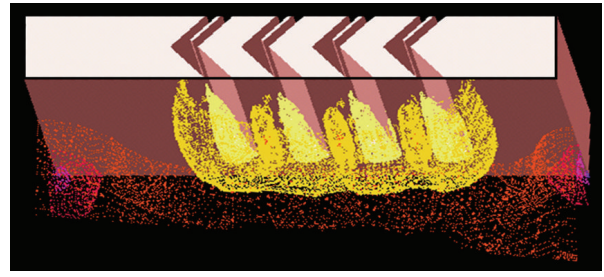

(b)

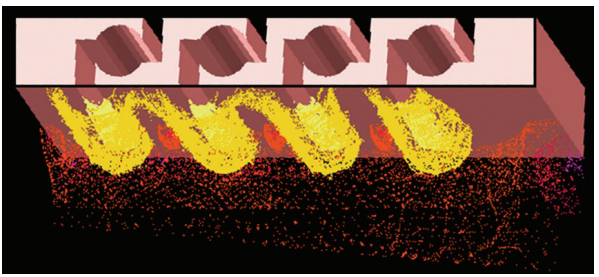

(d)

FIGURE 5: Transparency of the various slot structures to the RF magnetic field. The distribution of the RF electromagnetic energy density at the exit from slot due to (a) chevron, (b) chevron with a thin blade, (c) " $Z$-slot" structure, and (d) double L with cavity structure. 
due to the restriction of capacitive coupling to the plasma. The plasma extinction curve was not affected by the DB presence.

\subsection{Plasma Interaction with DB Structure}

3.3.1. Reduction of Capacitive Coupling from Antenna. Effective copper ionization occurs in a central area of the processing chamber and above the substrate [6]. The copper ions diffuse towards the substrate surface and walls and are accelerated by sheath voltage to the substrate surface. The range of ion energies after passing the sheath at the substrate was estimated from the electrical probe $\left(V_{\text {plasma }} \sim 36 \mathrm{~V}\right)$ and self-bias potential measurements at the substrate (observed within $+20 \mathrm{~V}>V_{\text {bias }}>-60 \mathrm{~V}$ for table power from $0 \mathrm{~W}$ to $1 \mathrm{~kW}$ ). Thus ion energy ranges from $\sim 16 \mathrm{eV}$ to $\sim 90 \mathrm{eV}$ based on choice of operating conditions. Metal transport occurs not only towards the substrate but also towards other internal hardware parts including the DB. Sheath geometry at the DB may be much more complex and will also depend on pressure, plasma density, electron temperature, and the electrical potential of the $\mathrm{DB}$. We provided good grounding conditions for $\mathrm{DB}$ to minimize its erosion and the impact of fluxes of charged species. Such conditions were required also for minimal capacitive coupling of the DB that might appear in series with ICP antenna and plasma. The RF electric field penetration was minimized due to slot's dimensions and no-line of sight through it. However, the experimental observations indicate that plasma can penetrate through the slots at certain conditions. This behavior was investigated by the sheath model described in the next section.

3.3.2. Sheath Model: Interaction with DB Structure. To investigate sheath properties, we developed sheath model under FEM commercial software [22]. The plasma sheath occupies a narrow domain between the plasma and the DB surface. Classic textbook references on plasma physics and sheath theory can be found elsewhere [23]. We used Lieberman's formulation for collisionless DC sheath. Utilizing the electrostatics from AC/DC module of implemented software, we computed the electric field within a sheath domain above the DB structure. Assumptions in the model were Maxwellian electrons at temperature $T_{e}$, cold ions $\left(T_{i}=0\right)$ in bulk domain, and quasi-neutrality of the plasma in bulk plasma $\left(n_{e}=n_{i}=n\right)$ and presheath $\left(n_{e s}=n_{i s}=n_{s}\right)$ domain. Boltzmann relation for electrons in sheath defines $n_{e}(x)$ as follows:

$$
n_{e}(x)=n_{e s} \exp \left(\frac{\Phi(x)}{T_{e}}\right)
$$

Sheath edge is considered at potential $\Phi=0$. From ion energy conservation, it follows that ion density within a sheath is described by the following relation:

$$
n_{i}(x)=n_{i s}\left(1-\frac{2 e \Phi(x)}{m_{i} u_{s}^{2}}\right)^{-1 / 2}
$$

The nonlinear equation governing the sheath potential and ion and electron densities is obtained by rewriting Poisson's equation after the substitution of relations (1) and (2) in form (here, the electron temperature is considered in electron volt units)

$$
\frac{d^{2} \Phi}{d x^{2}}=\frac{e n_{s}}{\varepsilon_{0}}\left[\exp \left(\frac{\Phi(x)}{T_{e}}\right)-\left(1-\frac{2 e \Phi(x)}{m_{i} u_{s}^{2}}\right)\right]^{-1 / 2} .
$$

The above procedure is easily implemented by GUI in Physics formulation of the multiphysics software. Here, we kept Poisson's equation in default form and in 3D space potential $\Phi(x, y, z)$ is coupled with variables $n_{e}(x, y, z)$ and $n_{i}(x, y, z)$ in form

$$
-\nabla \cdot \varepsilon_{r} \varepsilon_{0} \nabla \Phi=\rho=-e\left(n_{i}-n_{e}\right) .
$$

When defining subdomain expressions for the plasma region, we set conditions for ion density in form

$$
n_{i}= \begin{cases}n_{s} & \text { when } 2 \eta>1, \\ n_{s}(1-2 \eta)^{-1 / 2} & \text { when } 2 \eta<1,\end{cases}
$$

and electron density

$$
n_{e}= \begin{cases}n_{s} & \text { when } V>0 \\ n_{s} \exp \left(\frac{V}{T_{e}}\right) & \text { when } V<0\end{cases}
$$

Described conditions are governing numerical solution of Poisson's equation under assumption above. Symbols are $\eta=\mathrm{eV} /\left(m_{i} u_{s}^{2}\right)$, where $V$ is electric potential, $e$ is elementary charge, $m_{i}$ is ion mass, $n_{s}$ is plasma density at the sheath edge, and $u_{s}$ is Bohm velocity at the sheath edge. Symmetrical surface boundary conditions (BC) are set at the vertical sidewalls of each subdomain. The top surface boundary is set to relative plasma potential $V_{\text {plasma }}=26 \mathrm{~V}$, and the surface boundary at $\mathrm{DB}$ is set to ground potential. Interior boundaries are represented by continuity BC.

This sheath model is suitable for a one-dimensional study of sheath properties. Because we are investigating more complex geometry of plasma-surface interface, it may be less accurate. Specifically, its weakness may become evident at very high-density plasma when sheath size is small. This is due to the fact that (1) we are neglecting secondary electron emission from the exposed to plasma surface of the DB, and (2) we are assuming a constant bulk density over the computational domain including slots of the DB. Condition (1) may contribute to an increased plasma density inside an individual slot, especially when depositing copper films. An opposite effect is due to the loss of ions from plasma on the DB surface, which will result in reduced bulk density in dependence on the specific geometry of the slots, thus assumption (2) will not be fully satisfied.

In spite of these limitations, we used the model to investigate an impact of the plasma density on the penetration into DB's slots. Column (a) in Figure 6 indicates sheath size 


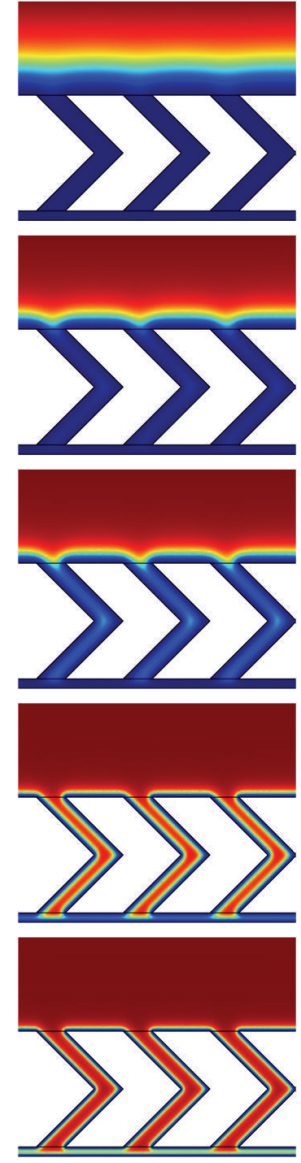

(a)

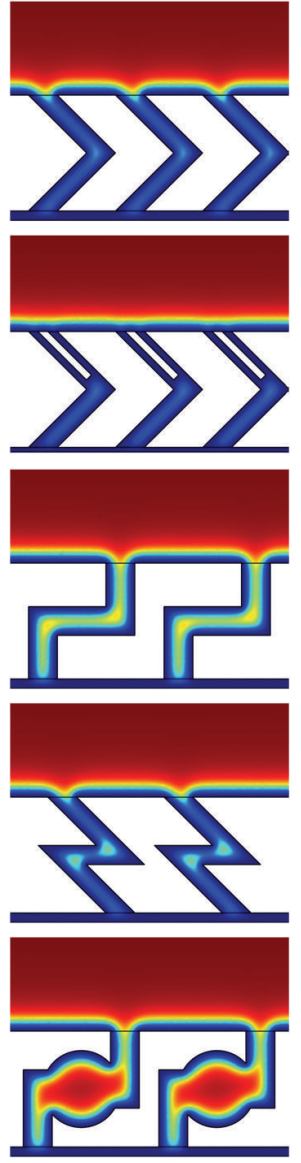

(b)
Figure 6: Simulation of the electron density by sheath model penetration of plasma into slots (a) at various plasma densities, and impact of the slot geometry (b) on plasma presence in the slot. The plots from top to down in column (a) represent simulation results at various bulk plasma density, that is, $1 \times 10^{9} \mathrm{~cm}^{-3}, 5 \times 10^{9} \mathrm{~cm}^{-3}$, $1 \times 10^{10} \mathrm{~cm}^{-3}, 5 \times 10^{10} \mathrm{~cm}^{-3}$, and $1 \times 10^{11} \mathrm{~cm}^{-3}$. Plots in column (b) are computed at bulk plasma density $1 \times 10^{10} \mathrm{~cm}^{-3}$.

and penetration of the plasma into a slot. The plots from top to bottom in column (a) represent simulation results at various bulk plasma densities: $1 \times 10^{9} \mathrm{~cm}^{-3}, 5 \times 10^{9} \mathrm{~cm}^{-3}$, $1 \times 10^{10} \mathrm{~cm}^{-3}, 5 \times 10^{10} \mathrm{~cm}^{-3}$, and $1 \times 10^{11} \mathrm{~cm}^{-3}$. Although the ICP core plasma density may be as high as $5 \times 10^{12} \mathrm{~cm}^{-3}$ [7], the plasma density close to deposition baffle applicable for use in the sheath model is lower. It follows from the sheath model that sheath thickness is smaller at increased bulk plasma density. Due to this, plasma can penetrate inside the chevron slot at densities above $1 \times 10^{10} \mathrm{~cm}^{-3}$.

The plots in column (b) of the Figure 6 indicate plasma penetration into various slot structures at the same pressure. Minimal penetration is in the case of chevron slot with blade (the 2 nd row) better than simple chevron slot (the 1st row). At a given pressure, plasma can penetrate more into " $Z$-slot" (the 4th row) than into "2L-slot" (the 3rd row). When an internal cavity is included as part of the slot-bottom plotplasma can be partially sustained inside the cavity due to increased bulk volume to internal surface ratio. This type of structure also exhibited easy plasma ignition comparing to the others.

3.4. Window Support, Thermal Performance, and Lifetime Duty. In this section, we will briefly mention other aspects of the DB in the IPVD apparatus. Image currents on the slats of the DB have to be minimized, so energy is not wasted in joule heating of the device slats. Currents in the DB may be considerable depending on the penetration of the plasma inside into the slots. Also, due to high plasma density, the $\mathrm{DB}$ is exposed to a substantial ion fluxes. As an illustration of such heat load during an early stage development, the damage produced by overheating, the DB in high-density metal-containing plasma is shown in Figure 7. In spite of excellent opacity to deposition, the chevron with blade was susceptible to failure at processing conditions.

Proper slot cooling must be provided to avoid DB damage. In the production version of the $\mathrm{DB}$, thermal stability is achieved by passing cooled water through the metal body portion of the slots. Full-power temperature rise at processing cycles is less than $30^{\circ} \mathrm{C}$. Current zero-failure $\mathrm{DB}$ in commercial tool is a result of the extensive simulation analysis, experimental tests, and compromise between many trades offs to satisfy all requirements mentioned above. In dependence of material and process conditions, the lifetime of DB in IPVD tool represents $250 \mathrm{kWh}$ to $800 \mathrm{kWh}$ between replacement or maintenance. With appropriate spacing between the dielectric window and the DB, we are able to achieve minimal species contamination on the window surface and to extend its lifetime.

\section{Conclusion}

In this work, diverse slot structures for an IPVD deposition baffle (used as a protection for the dielectric window of an ICP source) were described from an engineering standpoint. Several slot structure designs were proposed to satisfy the requirements (Section 3) of high RF magnetic field transparency, high opacity against deposition of conductive contamination on sensitive dielectric components of the ICP source, and equipment reliability. Various engineering aspects related to the deposition baffle are discussed. Over the course of the development, these structures were both experimentally and by simulation tools tested and optimized. Different deposition baffle slot structures were simulated by implementing a string model for deposition profile evolution, and through it on dielectric components of the ICP source and validated for an actual design. Among the many requirements, we focused on a specific structure of the slots and analysis to minimize deposition on the baffle. Transparency of the baffle to RF magnetic field is computed by electromagnetic field solver. Actual plasma density was practically insensitive to the slot type. Simple sheath model (FEM) was used to understand plasma interaction with slots in deposition baffle. Practical implementation of such deposition baffle in a commercial tool is a result of extensive simulation analysis, experimental tests, and engineering trade-offs needed to satisfy performance requirements. 


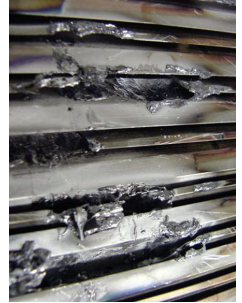

(a)

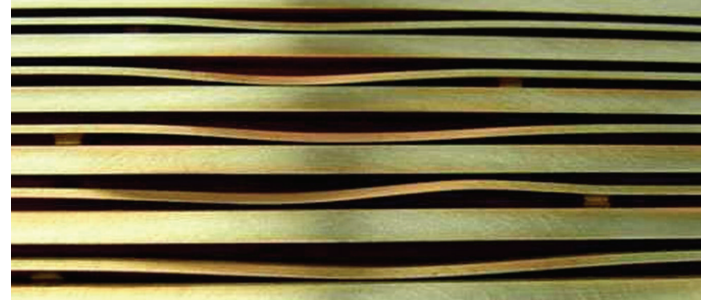

(b)

FIGURE 7: Failure of the chevron slot structure combined with thin blades, structure shown in Figure 4. Photo (a) shows overheated slots structure followed by slot's melting (Al), and (b) thin blade deformation due to the expansion as their temperature increased relatively to the temperature of a more robust and cooled chevron slat.

\section{Acknowledgments}

The authors would like to thank Turbo Horiuchi for motivation in this development work, James Grootegoed for technical assistance with preparation and running the experimental apparatus, and TEL designers and engineers (among the others Stephen Bachman and Hiroyuki Yokohara) for incorporating many features into design and helpful technical discussions. We would like to thank Eric Lee for careful revision of the paper.

\section{References}

[1] S. M. Rossnagel and J. Hopwood, "Magnetron sputter deposition with high levels of metal ionization," Applied Physics Letters, vol. 63, no. 24, pp. 3285-3287, 1993.

[2] J. Hopwood and F. Qian, "Mechanisms for highly ionized magnetron sputtering," Journal of Applied Physics, vol. 78, no. 2, pp. 758-765, 1995.

[3] S. M. Rossnagel, "Directional and ionized physical vapor deposition for microelectronics applications," Journal of Vacuum Science and Technology B, vol. 16, no. 5, pp. 2585-2608, 1998.

[4] S. M. Rossnagel, "Thin film deposition with physical vapor deposition and related technologies," Journal of Vacuum Science \& Technology A, vol. 21, no. 5, pp. S74-S87, 2003.

[5] J. A. Hopwood, Ed., Ionized Physical Vapor Deposition: Thin Films, Academic Press, San Diego, Calif, USA, 2000.

[6] J. Brcka, "Model-based ionized-PVD-source scaling and performance: hardware-feasibility study," IEEE Transactions on Plasma Science, vol. 35, no. 3, pp. 626-636, 2007.

[7] J. Brcka, "Simulation and characterization of IPVD system with external ICP antenna," Plasma Processes and Polymers, vol. 4, no. 1, pp. S952-S959, 2007.

[8] J. S. Drewery, G. Reynolds, D. A. Russell et al., "Method and apparatus for ionized physical vapor deposition," US Patent no. $6,287,435,2001$.

[9] M. J. Grapperhaus, Z. Krivokapic, and M. J. Kushner, "Design issues in ionized metal physical vapor deposition of copper," Journal of Applied Physics, vol. 83, no. 1, pp. 35-43, 1998.

[10] S. Rauf, P. L. G. Ventzek, and V. Arunachalam, "Ionized physical vapor deposition of $\mathrm{Cu}$ on $300 \mathrm{~mm}$ wafers: a modeling study," Journal of Applied Physics, vol. 89, no. 5, pp. 2525-2534, 2001.

[11] K. Tao, D. Mao, and J. Hopwood, "Ionized physical vapor deposition of titanium nitride: a global plasma model," Journal of Applied Physics, vol. 91, no. 7, p. 4040, 2002.
[12] V. Vyas and M. J. Kushner, "Scaling of low-pressure ionized metal PVD reactors," in Proceedings of the AVS 51st International Symposium, pp. 14-19, Anaheim, Calif, USA, November 2002.

[13] P. J. Stout, D. Zhang, S. Rauf, and P. L. G. Ventzek, “Comparing ionized physical vapor deposition and high power magnetron copper seed deposition," Journal of Vacuum Science and Technology B, vol. 20, no. 6, pp. 2421-2432, 2002.

[14] J. Brcka, "Process apparatus and method for improving plasma distribution and performance in an inductively coupled plasma," US Patent no. 6,237,526, 2001.

[15] J. Brcka and R. L. Robison, "Wafer redeposition impact on etch rate uniformity in IPVD system," IEEE Transactions on Plasma Science, vol. 35, no. 1, pp. 74-82, 2007.

[16] R. A. Powell and S. M. Rossnagel, Eds., PVD for Microelectronics: Sputter Deposition Applied to Semiconductor Manufacturing: Thin Films Vol. 26, Academic Press, San Diego, Calif, USA, 1999.

[17] J. Brcka, "Modified transfer function deposition baffles and high density plasma ignition therewith in semiconductor processing," US Patent no. 6,946,054, 2005.

[18] J. Brcka, M. Kleshock, and T. Provencher, "Cooled deposition baffle in high density plasma semiconductor processing," US Application Publication 2004/0129221, 2004.

[19] J. Brcka, J. Parizek, J. Breza, and I. Jelemensky, "Simulator of vertical profiles in technology of integrated circuits," Journal of Electrical Engineering, vol. 2, pp. 33-41, 1992.

[20] M. J. Grapperhaus, “Internal materials ," TEL Arizona.

[21] Ansoft Inc., (currently product of ANSYS, Inc.), http://www .ansys.com/ .

[22] Multiphysics Comsol, Inc., http://www.comsol.com/ .

[23] M. A. Lieberman and A. J. Lichtenberg, Principles of Plasma Discharges and Materials Processing, John Wiley \& Sons, New York, NY, USA, 1994. 

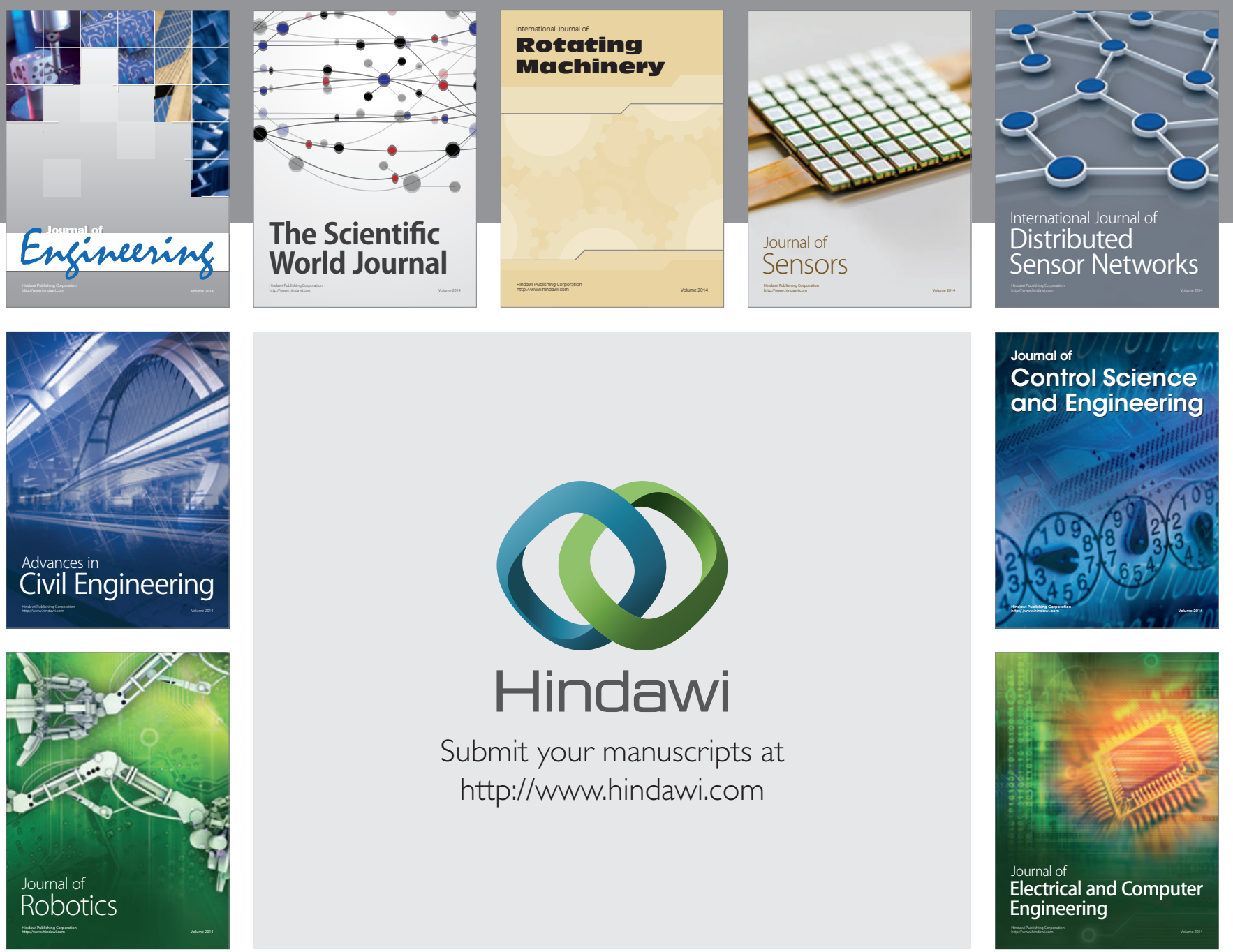

Submit your manuscripts at

http://www.hindawi.com
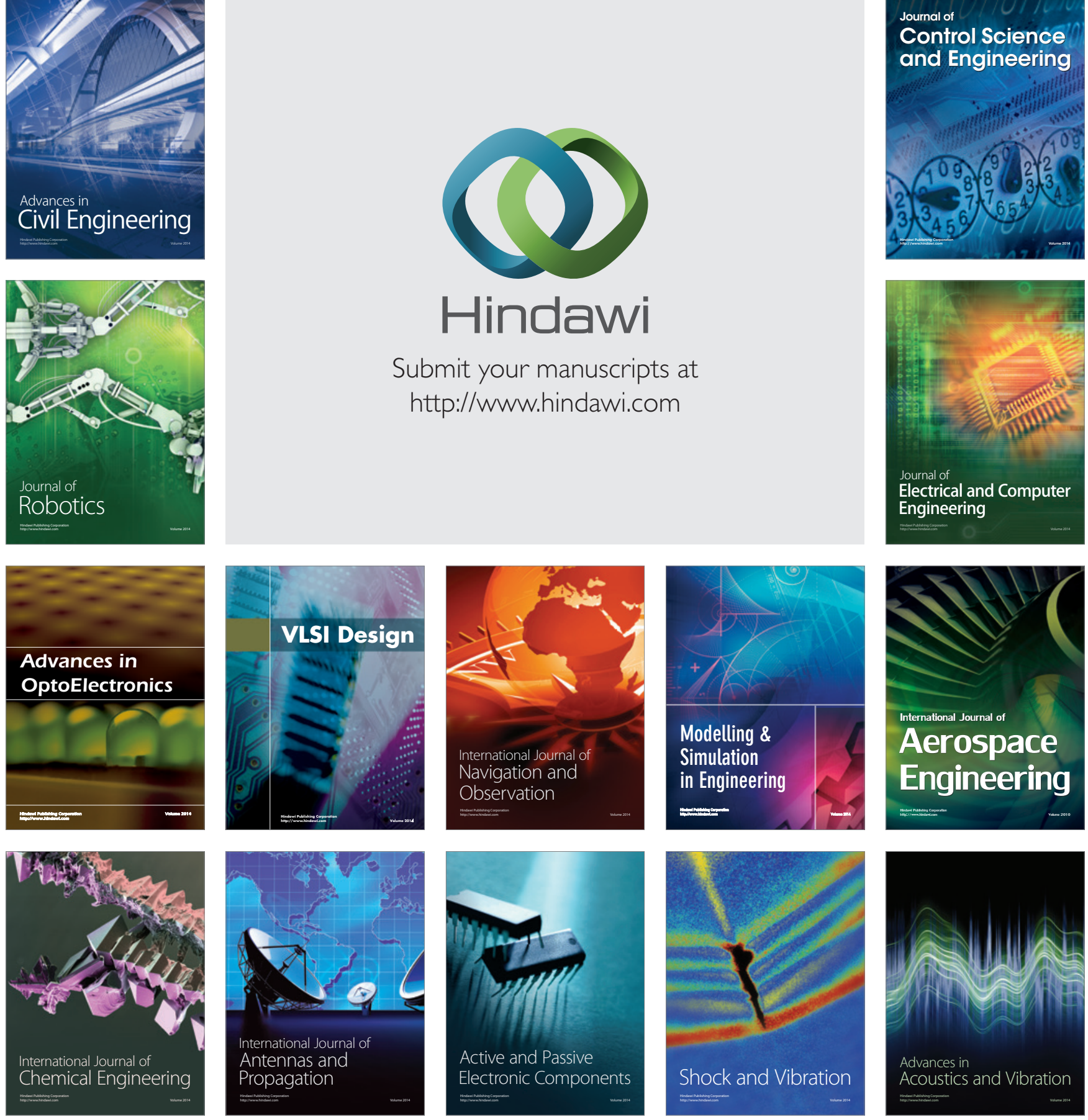